\title{
Encrucijadas sociales de la innovación ${ }^{*}$ Social crossroads of innovation
}

\author{
José A. López Cerezo y MARTA I. GonZÁLez \\ Universidad de Oviedo
}

RESUMEN. La creciente literatura aparecida en los últimos años sobre innovación ha producido multitud de definiciones y clasificaciones de la misma en las que se reflejan disciplinas y tradiciones de origen, así como compromisos teóricos y prácticos. Distinguir entre innovación de productos o innovación de procesos, o diferenciar de acuerdo con el grado de novedad de los resultados de la innovación (o la "intensidad" innovadora) son algunas de estas estrategias taxonómicas. Aunque los enfoques más clásicos sobre innovación han obviado sistemáticamente los aspectos sociales de la misma, recientemente ha aparecido una nueva categoría, la "innovación social", etiqueta que engloba clases muy distintas de prácticas. En esta contribución analizaremos los distintos sentidos en los que se entiende la "innovación social" y defenderemos que una innovación apropiada ha de ser siempre una forma de innovación social.

Palabras clave: innovación, innovación social, entorno social, política científica
ABSTRACT. Recent and growing literature on innovation has produced a good number of definitions and categorizations that reflect their disciplines and approaches of origin, as well as their theoretical and practical commitments. The distinction between product innovation and process innovation, or the classification according to the degree of novelty produced (or innovation "intensity"), are among the taxonomical strategies. Although classical approaches on innovation have systematically ignored its social aspects, the new and successful category of "social innovation" constitutes a label that includes very diverse types of practices. In this contribution we will analyze the different understandings of "social innovation" and we will argue that an appropriate innovation should always be a kind of social innovation.

Key words: Innovation, Social Innovation, Social Milieu, Science Policy

La innovación está de moda. Junto con otros conceptos como el de "sociedad de la información", la innovación es un huésped permanente del discurso de políticos, empresarios, sindicalistas y los más variados agentes sociales. Aunque no es raro escuchar que se trata de un mero cambio de etiqueta, de una moder-

\footnotetext{
* Este trabajo ha sido posible gracias al proyecto "Políticas de la cultura científica" (MICINN-12-FFI2011-24582).
} 
nización en los términos, para hacer referencia a lo mismo de siempre, a saber, el sistema de ciencia y tecnología, no compartimos esa opinión.

Los significados de las palabras tienen cierta magia, pues son el vehículo para que estas no sólo representen la realidad sino que también la transformen. Los cambios terminológicos suelen ser indicativos de cambios en las filosofías subyacentes, manifestando además normalmente un compromiso con esos mismos presupuestos. Cuando las palabras que cambian son las que hacen referencia al sistema de ciencia y tecnología, lo que esos cambios registran es la evolución de las culturas políticas al respecto. Es lo que, en nuestra opinión, está ocurriendo actualmente con la obsesión por la innovación cuando se aborda el tema de la ciencia y la tecnología.

Sin embargo, por desgracia es frecuente encontrar una comprensión muy limitada del fenómeno de la innovación, una comprensión que desatiende la relevancia de sus dimensiones sociales y el papel potencial de la innovación como servicio público. Si se examinan los programas públicos de estímulo de la innovación, en el marco de los sistemas de ciencia y tecnología a nivel nacional o regional, estos parecen capítulos de la política económica expresados también en el lenguaje de la competitividad y la rentabilidad. La crisis económica actual ha agudizado aún más esta tendencia y legitimado que los sistemas de ciencia y tecnología tengan como objetivo y criterio principal de evaluación su productividad económica.

Aunque los enfoques más clásicos sobre innovación han obviado sistemáticamente los aspectos sociales de la misma, recientemente ha aparecido una nueva categoría, la "innovación social", etiqueta que engloba clases muy distintas de prácticas. En esta contribución ensayaremos una reflexión crítica de naturaleza ético-política sobre el fenómeno de la innovación y sobre el énfasis en la innovación de las actuales políticas públicas sobre ciencia y tecnología. Analizaremos los distintos sentidos en los que se entiende la "innovación social" y defenderemos que una innovación apropiada ha de ser siempre una forma de innovación social. A través de la ilustración de la relevancia de los aspectos sociales de la innovación discutiremos las consecuencias de esta perspectiva para el significado de la promoción de una "cultura de la innovación" o una "sociedad de la innovación". A fin de situar el actual estado de cosas, comenzaremos esbozando una breve perspectiva histórica del proceso por el que la política científica se ha convertido en política de innovación en España.

\section{Ciencia, tecnología e innovación en perspectiva histórica}

Después de la muerte de Franco y el fin de la dictadura, a finales de los años 70, en España no existía propiamente una política de estado en materia de ciencia 
y tecnología. El marco legal con el que arranca esa política no llega hasta el ingreso de España en la Unión Europea a mediados de los años 80. En 1986 se hizo pública la Ley del Fomento y Coordinación General de la Investigación Científica y Técnica (Ley 13/1986 de 14 de abril), más conocida como la "Ley de la Ciencia". En ella se crean los principales instrumentos para promover y organizar el hasta entonces disperso y poco eficiente esfuerzo de las universidades y administraciones públicas españolas en materia de ciencia y tecnología.

Se crea por ejemplo la Comisión Interministerial de Ciencia y Tecnología (CICYT) para coordinar las actividades de diversos ministerios con competencias en ciencia y tecnología, y dentro de la CICYT se crea la Agencia Nacional de Evaluación y Prospectiva (ANEP), como órgano evaluador de los proyectos de investigación y las actividades que se enmarcan en el ámbito de competencia de la CICYT.

Además se establecen planes plurianuales para la promoción de la ciencia y la tecnología, cuya programación y seguimiento corresponde a la CICYT. Esos planes, de los que se desarrollaron tres entre 1988 y 1999, son los conocidos como Planes Nacionales de Investigación Científica y Desarrollo Tecnológico (Planes Nacionales de I+D). En ellos se crearon programas nacionales de formación de investigadores a través de la convocatoria de becas, programas sectoriales de apoyo a la investigación en diversos ámbitos, etc., así como mecanismos de apoyo a la transferencia de conocimientos al ámbito del sistema productivo (a través del Centro para el Desarrollo Tecnológico e Industrial, dependiente del Ministerio de Industria - reforzado por otros mecanismos de interfase como la red OTRI de transferencia de resultados de investigación). ${ }^{1}$

Se trataba en general de planes de $\mathrm{I}+\mathrm{D}$ que, de acuerdo con su justificación explícita, tenían como objetivo último promover el llamado sistema "cienciatecnología-industria". Eran planes que reflejaban así en cierta medida la filosofía del viejo modelo lineal de desarrollo apadrinado por Vannevar Bush. ${ }^{2}$ De acuerdo con este modelo, el bienestar de las naciones depende de su desarrollo económico, un desarrollo que se edifica sobre la producción industrial. Y, a su vez, el mayor y más poderoso estímulo para la industria procede de la innovación tecnológica, la cual es básicamente una cuestión de oferta científica.

${ }^{1}$ Véase, en general, la sección correspondiente a España, firmada por J. Sebastián (2000a), en la "Guía Iberoamericana de la Administración Pública de la Ciencia", en www.campusoei.org/guiaciencia/espana.htm

${ }^{2}$ El texto clásico de V. Bush es el informe presidencial Science - The Endless Frontier, de julio de 1945, reimpreso por la National Science Foundation (NSF) en 1980 - la misma organización que Bush recomendó crear en 1945 para garantizar la regulación autónoma de la integridad y la productividad de la investigación científica. La NSF es creada cinco años después, en 1950 . 
La tarea de los gobiernos, a este respecto, sería fundamentalmente la provisión de recursos materiales y humanos para la investigación y, corrigiendo el modelo lineal puro de Bush, priorizar líneas estratégicas de I+D y articular los medios legales y administrativos que, como tratan de hacer la legislación y los mecanismos de interfase, aseguren el correcto funcionamiento del sistema "ciencia-tecnología-industria" y el aprovechamiento de la inversión.

En la segunda mitad de los años 90 se produce un cambio de gobierno del que resulta una reforma del sistema de ciencia y tecnología heredado del anterior ejecutivo. A finales de los años 90 (Ley 50/1998) se crea un nuevo organismo público como "órgano de apoyo" (o control, según algunos) a la CICYT, la Oficina de Ciencia y Tecnología (OCYT), dependiente directamente de Presidencia del Gobierno. ${ }^{3}$ En el contexto de la justificación de la reforma del sistema, se entona entonces un "mea culpa" institucional acerca de las limitaciones de los viejos planes plurianuales para incentivar la innovación.

No se trataba de un problema de volumen o calidad de la investigación. No había dudas del éxito de esos planes de I+D en términos de indicadores de producto, pues en una década se consiguió elevar enormemente el volumen y calidad de la investigación científica española hasta disminuir significativamente la distancia que la separaba de la media europea (pese a consumir anualmente solo alrededor de un $0,8 \%-0,9 \%$ del PIB) y hasta alcanzar a finales de la década de los 90 al conjunto de América Latina en términos de número de artículos recogidos en la base de datos del Science Citation Index. ${ }^{4}$ Sin embargo, a pesar de tal éxito, se apreció un importante déficit en la transferencia de conocimientos científicos y desarrollos tecnológicos al sistema productivo. Los indicadores de innovación, frente a la referencia inevitable de Estados Unidos, no respondían al potencial de la I+D española (que en 1999 alcanzó ya el 1\% del PIB según datos oficiales de la OCYT). ${ }^{5}$

En noviembre de 1999, el Gobierno nacional aprobó un nuevo plan que trata de corregir ese estado de cosas. Se trata de un plan que añade la palabra "innovación" al acrónimo tradicional I+D, pasando a llamarse "Plan Nacional de I+D+I" 2000-2003. Y, corrigiendo un cierto sesgo decimonónico, el plan ya no tiene como objetivo promover el sistema "ciencia-tecnología-

${ }^{3}$ En esta misma reforma legal se otorga la presidencia de la CICYT al Presidente del Gobierno.

${ }^{4}$ Los datos comparativos entre España y Latinoamérica pueden encontrarse en la página web de la Red Iberoamericana/Interamericana de Indicadores de Ciencia y Tecnología (RICYT): www.ricyt.edu.ar

${ }^{5}$ Todos los datos e información oficial sobre el sistema español de ciencia y tecnología está disponible y es fácilmente accesible en Internet. Véase, en particular, http://www.idi.mineco.gob.es/portal/site/MICINN/ 
industria" sino el sistema "ciencia-tecnología-empresa", no restringiendo así el sector productivo a las actividades industriales (para dar entrada a las actividades en el sector de servicios, telecomunicaciones, etc.). España incorporaba así una tendencia general en los países de su entorno europeo: el énfasis en la innovación. ${ }^{6}$

En abril del 2000, la OCYT se disolvió con motivo de la creación de un nuevo Ministerio de Ciencia y Tecnología. Ello supuso la reorganización general del sistema, ${ }^{7}$ y también un cambio en los estilos políticos para dar un gran peso a las consideraciones de política económica en materia de ciencia y tecnología. Un nuevo cambio de gobierno en 2004 llevó la ciencia de vuelta al terreno de la educación, separándola otra vez de la innovación y la economía. No obstante, la nueva legislatura socialista que dio comienzo en 2008 se rindió definitivamente a la tendencia general con la creación del Ministerio de Ciencia e Innovación, haciendo explícito de un modo muy claro el patrón general. Las dudas con respecto a dónde situar las universidades dan cuenta de las dificultades conceptuales de compatibilizar la doble misión de estas en el nuevo escenario: por un lado, la vocación de servicio público en educación, por otro, las nuevas alianzas y obligaciones productivas. El Ministerio de Ciencia e Innovación incluyó las universidades en un principio, pero solo un año después, las competencias en materia de educación universitaria habían regresado al nuevo Ministerio de Educación, Política Social y Deporte. Recientemente, en 2102 y en el contexto de la grave crisis social y económica que afecta a España, el nuevo gobierno popular ha completado la transición de la ciencia a la innovación colocando la Secretaría de Estado de Investigación, Desarrollo e Innovación dentro del Ministerio de Economía y Competitividad. Poco antes, a mediados de 2011, se había aprobado la nueva Ley de Ciencia, Tecnología e Innovación, con la que el Sistema de Ciencia y Tecnología pasa a denominarse oficialmente "Sistema de Ciencia, Tecnología e Innovación".

Además, y con poco tiempo de demora, el añadido de la innovación fue una iniciativa que está teniendo un efecto multiplicativo en otros niveles administrativos. En la ordenación de sus propias políticas de ciencia y tecnología, los gobiernos autonómicos han ido también orientado explícitamente dichas polí-

\footnotetext{
${ }^{6}$ En EE.UU. hallamos nuevamente los orígenes de la intervención gubernamental para garantizar la productividad de la inversión en $\mathrm{I}+\mathrm{D}$, corrigiendo así desde la esfera pública la autonomía del sistema presupuesta por el modelo lineal. Es este sentido destacan una serie de leyes creadas a principios de los años 80 , sobre innovación tecnológica, transferencia de tecnologías, patentes y marcas registradas, que trataban de promover el flujo de resultados entre el sistema de producción de conocimiento y el sistema de producción de bienes y servicios. Véase Guston (2000).

${ }^{7}$ Su estructura es descrita en B.O.E. de 13-5-2000, pp. 17663 ss.
} 
ticas hacia el sistema "ciencia-tecnología-innovación". En Cataluña, por ejemplo, está en vigor el Plan de Investigación e Innovación (2010-2013), y las competencias de universidades e investigación se encuentran juntas en el Departamento de Economía y Conocimiento. Por su parte, en el País Vasco la apuesta por la innovación es fuerte, con la creación en 2007 de Innobasque, ${ }^{8}$ la Agencia de la Innovación encargada de impulsar el Sistema Vasco de Ciencia, Tecnología e Innovación con el objetivo de "convertir a Euskadi en el referente europeo en materia de innovación".

\section{El significado de la innovación}

El énfasis en la innovación nos ha llevado así de la industria a la empresa en la orientación del gasto público en materia de ciencia y tecnología. Enfatizar la innovación, y pasar de la industria a la empresa, es un paso muy importante, si lo que nos interesa es obtener beneficios desde el sistema I+D en el ámbito de la competitividad y el crecimiento económico. La situación económica del país parece así requerirlo. Sin embargo, se trata de un paso aún insuficiente. Al igual que la industria es una parte del ámbito empresarial, la empresa es sólo una parte de la sociedad, en el Estado español y en sus distintas autonomías.

En nuestra opinión, al perfilar los objetivos del esfuerzo en I+D desde la política pública, más que del sistema "ciencia-tecnología-empresa" deberíamos hablar del sistema "ciencia-tecnología-sociedad", sentido amplio, incluyendo por supuesto al sector empresarial, quien puede y debe beneficiarse del esfuerzo público en ciencia y tecnología. No es solo una cuestión de elegir mejor las palabras o expresiones al definir un planteamiento político; no es una mera cuestión terminológica.

¿Por qué ese objetivo, el de orientar la ciencia y la tecnología al bienestar de la sociedad, no está garantizado con el solo estímulo de la innovación? ¿Cómo se relaciona la innovación con el mercado, la sociedad y el sistema de ciencia-tecnología?

Un hecho evidente, como se mencionaba al comienzo, es que la innovación está de moda. En España, la ciencia se ha unido a la innovación en la denominación de ministerios, secretarías de estado, consejerías o departamentos. Los planes plurianuales son planes de investigación e innovación. Los consultores de innovación están desbordados por tanto trabajo. Todo el mundo habla hoy

\footnotetext{
${ }^{8} \mathrm{http}: / /$ www.innobasque.com

${ }^{9}$ En un sentido análogo, J. Sebastián (2000b) hace referencia al sistema "ciencia-tecnología-innovación-gobernabilidad", añadiendo que la clave de este último eslabón es la satisfacción de las demandas sociales.
} 
de innovación y emprendimiento como las tablas de salvación de una situación cada vez más preocupante; sin embargo no siempre está claro a qué se hace referencia con el término.

No puede, en particular, confundirse la innovación con la ciencia o la tecnología, o incluso con la alianza de estas. La innovación se edifica normalmente sobre el desarrollo científico-tecnológico pero no se deriva inexorablemente de la ciencia y la tecnología. Con una inversión similar en términos de porcentaje de PIB, la producción científica europea sobrepasó en los años 90 a la norteamericana, sin embargo Europa está aún lejos de la capacidad innovadora de EE.UU, por patentes registradas y por transferencia de tecnología desde el laboratorio a la empresa. Es lo que se ha denominado, de un modo un tanto pretencioso, la "paradoja europea". ${ }^{10}$

¿Qué es entonces la innovación? La creciente literatura aparecida en los últimos años sobre innovación ha producido multitud de definiciones y clasificaciones de la misma en las que se reflejan disciplinas y tradiciones de origen, así como compromisos teóricos y prácticos. Distinguir entre innovación de productos o innovación de procesos, o diferenciar de acuerdo con el grado de novedad de los resultados de la innovación (o la "intensidad" innovadora) son algunas de estas estrategias taxonómicas. Una definición estándar, como la proporcionada por la tercera edición del Manual de Oslo (OECD, 2005: 33) caracteriza la innovación como "la implementación de un producto (bien o servicio) o proceso nuevo o con un alto grado de mejora, o un método de comercialización u organización nuevo aplicado a las prácticas de negocio, al lugar de trabajo o a las relaciones externas". A partir de esta base, se diferencian habitualmente las innovaciones incrementales (basadas en conocimiento al uso) de las innovaciones radicales que generan breakthroughs en el modo de hacer las cosas.

Un enfoque teórico habitual en los estudios sobre innovación es el de los sistemas nacionales de innovación (con adaptaciones para sistemas regionales o sectoriales), ${ }^{11}$ con autores como C. Freeman, B. Lundvall, R. Nelson y M. Porter. En estos enfoques suele asumirse la teoría de sistemas para analizar la red de instituciones (industrias, universidades, departamentos del gobierno, etc.) cuyas actividades e interacciones determinan el comportamiento innovador de las empresas de un territorio o de un sector productivo. Se supone que la com-

${ }^{10}$ Sin embargo, todo debe decirse, muchos de los avances científicos o políticos que han repercutido en una mejora de la calidad de vida, sí tienen su origen en el viejo mundo.

${ }^{11}$ Otro término habitual es el de "cluster", entendido como un "grupo geográficamente denso de empresas e instituciones pertenecientes a un campo tecnológico concreto" (Porter, 2003). 
petitividad de una empresa está asociada a su capacidad innovadora, es decir, a su capacidad para desarrollar o apropiarse de nuevos conocimientos económicamente útiles. Como acertadamente ha señalado el físico español Emilio Méndez, ${ }^{12}$ la innovación requiere dos cosas unidas: novedad y beneficio económico. Hay por tanto dos conceptos clave involucrados en este modo de entender la innovación: la novedad y la rentabilidad (potencial). ${ }^{13}$ Lo cual es ya interesante puesto que, aunque la innovación está habitualmente asociada al desarrollo tecnológico o la apropiación de conocimiento científico por parte de las empresas, innovar no involucra necesariamente a la ciencia y la tecnología, como ya recoge también la última edición del Manual de Oslo. Algunas innovaciones españolas, como la fregona o el chupa-chups (caramelo con palo), o la revolución en los mercados introducida por las empresas de comida rápida, son buenos ejemplos de ello. ${ }^{14}$ Hablaremos pues de la innovación tecnoproductiva.

En su documento sobre Sistemas Nacionales de Innovación, la OCDE (1997) criticaba los enfoques tradicionales limitados a contabilizar inputs (en forma de inversión) y outputs (en forma de patentes) para el análisis y la evaluación de las políticas tecnológicas. Frente a esta aproximación, se defendía la importancia de centrar los análisis en las interacciones entre los distintos agentes e instituciones implicados en la conversión de los inputs en outputs. La innovación, se argumenta, "es el resultado de un complejo conjunto de relaciones entre actores que producen, distribuyen y aplican varios tipos de conocimiento" (OCDE, 1997: 9). Entre la lista de actores, se cita a las empresas privadas, las universidades, las instituciones de investigación públicas y las personas que trabajan en ellas. Lo importante, insisten, es la red de interacciones. Dado este planteamiento, el reto entonces es dinamizar estas interacciones a través de incentivos financieros o mecanismos de interrelación (llamados también de interfase) que, como las oficinas de transferencia de resultados de investigación, tratan de ajustar la oferta de conocimiento a la demanda por parte del sistema de producción de bienes y servicios.

12 “Innovación: mitos y realidad", El País, 12-4-2000, p. 44. Véase también el llamado Manual de Oslo, documento de la OECD de indicadores normalizados de innovación (OECD, 2005); así como su adaptación al contexto latinoamericano: Manual de Bogotá, Bogotá: OEA, 2000.

${ }^{13}$ Algunos autores como C. Freeman no añaden la cualificación "potencial", dando al éxito un carácter definitorio y, en los casos de falta de rentabilidad, hablando simplemente de "intentos de innovación fracasada".

${ }^{14}$ Una innovación puede también limitarse a la fase de comercialización, al abrir por ejemplo nuevos mercados, o bien afectar a estrategias comerciales, o de naturaleza administrativa o financiera, etc. 
Una primera cosa que llama la atención de este enfoque es su omisión de los agentes sociales no directamente involucrados en actividades de I+D (investigación y desarrollo) o vinculados al mundo de la empresa o las finanzas. Sin embargo, la viabilidad de un producto innovador o incluso de una línea de innovación sectorial no solo depende de las empresas, las instituciones donde se desarrolla la investigación, sus relaciones y un adecuado respaldo financiero. Esta es una red que parece alimentarse a sí misma. La viabilidad de la innovación depende en última instancia de un contexto social receptivo y favorable, en el cual se valore un resultado como algo nuevo y de interés. En este sentido, el éxito de la innovación (o incluso que sea contemplada como tal) depende ante todo de que los consumidores o los receptores sociales directos de la innovación respondan favorablemente a la misma. De otro modo los productos innovadores perecen de muerte natural. La novedad y la rentabilidad no son por tanto rasgos esenciales de productos o procesos sino que dependen de contextos sociales dados, lo que en la literatura sociológica se llama "anillos de creencia y acción”. Barry Barnes (1988) distingue entre: objetos materiales de la epistemología popular, objetos materiales contextualmente dependientes, objetos sociales con referente material y objetos sociales con marcador material. El caso de la innovación es de los objetos sociales con referente material. Un producto innovador, como una joya o una mala hierba, tiene por supuesto un referente material que, como en el caso de las sillas o las mesas de la epistemología popular no depende de sujetos cognoscentes o agentes sociales para tener cierta forma, textura, etc. Pero no es solo eso. Que un caramelo con un palo constituya una innovación, o un cristal una joya, depende de un anillo de creencia y acción de naturaleza social, de que los consumidores y otros agentes sociales relevantes crean lo que creen y se comporten como de hecho lo hacen.

Pero no sólo los consumidores forman parte de ese "entorno social" frecuentemente omitido al hablar de sistemas de innovación. Hay otras categorías sociales implicadas igualmente relevantes, quizás en ocasiones más difusas pero no menos reales. Se trata de los públicos afectados o interesados en la innovación, sea por constituir consumidores potenciales (por ejemplo de servicios médicos o productos farmacéuticos), por ser afectados debido a consecuencias colaterales de la innovación (como la población expuesta al incremento del paro en una zona a causa de la automatización de una industria) o por constituir un público interesado debido a motivos políticos o ideológicos (por ejemplo los miembros de organizaciones ecologistas o asociaciones de consumidores). Cada vez con más fuerza, y no solo en países desarrollados, el éxito de la innovación depende de la reacción favorable, o al menos no hostil, de estas y otras categorías sociales. El innovador proyecto para construir el 
primer parque eólico marítimo en España en el Mar de Trafalgar fracasó en 2005 por la oposición de grandes sectores de la sociedad gaditana que vieron en él una amenaza para el turismo y las pesca (Todt, González y Estévez, 2011). Una innovación como la producción de carne sintética a partir de células madre, presentada como solución potencial para dar respuesta a los problemas éticos y ambientales de la industria ganadera, se enfrenta desde su origen a la posibilidad de un fracaso debido al rechazo de los consumidores. Mark Post, director del equipo de la Universidad de Maastricht que lleva a cabo la investigación, al mismo tiempo que declaraba recientemente en Madrid estar a un paso de conseguir la primera hamburguesa "cultivada", reconocía que muy pocas personas estarían dispuestas a comerla. ${ }^{15} \mathrm{Si}$ esta carne convertida finalmente en producto de consumo a precio razonable fuera rechazada por los consumidores, se trataría de un caso claro de fracaso de un producto innovador en el eslabón de la comercialización debido a motivos que nada tienen que ver con factores de naturaleza técnica o económica. Una percepción pública negativa puede ser un obstáculo formidable para la innovación.

Es más, la sensibilidad institucional respecto al financiamiento de la $\mathrm{I}+\mathrm{D}$ o la percepción empresarial de la necesidad de innovar, dependen crecientemente del apoyo de la ciudadanía a la ciencia, la tecnología y la innovación, de la extensión de una cultura científica que genere una percepción favorable y respaldo social. No tener en cuenta el "entorno social", complejo ya en sí mismo, es descuidar un factor clave para la génesis y consolidación de los sistemas de innovación.

Paradójicamente, no es infrecuente en la literatura encontrar el reconocimiento de que los procesos de innovación deberían ser sensibles al contexto. Por ejemplo, se admite generalmente que la transferencia tecnológica a la empresa debe tener en cuenta las especificidades locales y sectoriales, por un lado, y la dependencia de dinámicas globales, por otro. El problema es seguir manteniendo una visión muy restrictiva de eso que llamamos "el contexto". Otra faceta de la sensibilidad contextual de esos procesos tiene que ver con el contexto social, configurado por una red heterogénea de actores, donde tiene lugar la apropiación social de la innovación. Un conocido ejemplo de la multidimensionalidad de un fenómeno, con un carácter constitutivamente social, lo ofrece el tema del riesgo. Como muestran los casos de la energía nuclear o las vacas locas, la adecuada gestión del riesgo en la sociedad contemporánea requiere que este no sea reducido a las variables técnicas y económicas tradicionales. La aceptabilidad del riesgo no depende únicamente de la probabilidad

\footnotetext{
${ }^{15}$ Público, 23/01/2013.
} 
de ocurrencia de un daño y la magnitud de este, ni de un cálculo económico que estime la probabilidad anual de fatalidad para un colectivo dado, pues hay otros muchos factores que contribuyen a personalizar los peligros y son la base para las pautas sociales de aceptabilidad: por ejemplo la posibilidad de compensación, su naturaleza catastrófica, la voluntariedad en la exposición, la equitatividad en su distribución, etc. Del mismo modo, también la innovación es constitutivamente social, un producto social con referente material, y su destino se encuentra sometido a factores que trascienden los entornos científicotecnológicos y económicos.

\section{De la innovación social a la sociedad de la innovación}

En los últimos años, sin embargo, e intentando superar las limitaciones de los enfoques tradicionales, los estudios sobre innovación se han vuelto hacia la nueva categoría de "innovación social". La etiqueta "innovación social" se ido popularizando, hasta el punto de que un reciente artículo (Edwards-Schachte et al. 2012) analizaba hasta 76 definiciones distintas. Frente a la idea tradicional de la innovación orientada al mercado, la "innovación social" se referiría a aquellas innovaciones que quedan fuera de este ámbito. Es decir, por innovación social se entiende, en términos generales, la innovación con fines sociales. Tal y como señala Javier Echeverría (2008), la última edición del Manual de Oslo sigue contemplando únicamente las innovaciones en el sector empresarial, aunque reconoce el interés de la innovación en ámbitos públicos (como pueden ser la educación o la salud). De acuerdo con Echeverría, mientras que la innovación tradicionalmente entendida se refiere a valores económicos y empresariales, la "innovación social" se referiría a valores sociales o culturales (bienestar, salud, calidad de vida, nivel cultural o educación). En su concepción axiológica de la innovación las diferentes categorías hacen referencia a los distintos valores a los que se orientan las innovaciones. De un modo similar, Mulgan et al. (2007) definen la innovación social como: "actividades y servicios innovadores que surgen con el objetivo de satisfacer alguna necesidad social y que son desarrollados y difundidos predominantemente por organizaciones cuyos propósitos primarios son sociales".

La innovación social entendida de este modo como innovación orientada hacia los valores sociales, puede promoverse desde diferentes ubicaciones. Típicamente, sus fuentes principales están en el sector público (salud, educación, servicios sociales) u organizaciones no gubernamentales y movimientos de base, como señalan Mulgan et al. (2007); pero también iniciativas empresariales, como las relacionadas con la alimentación ecológica o el comercio justo, podrían entenderse claramente como innovaciones sociales. Este tipo de ini- 
ciativas delimitan un nuevo territorio híbrido entre lo público y lo privado, lo que tiene y lo que no tiene ánimo de lucro. Este espacio, cada vez más poblado con empresas con fines sociales, se ha identificado recientemente como "cuarto sector" (Sabeti, 2009).

Además de relacionarse con los objetivos sociales, la innovación social se asocia frecuentemente con iniciativas que parten de la propia sociedad: desde la capacidad de innovar de los usuarios al apropiarse de los productos tecnocientíficos disponibles en el mercado hasta las formas colaborativas de creación de conocimiento e innovaciones, como las que se han hecho comunes en el desarrollo de software libre, que reunirían a la vez innovaciones en los procesos y en los productos (Núñez Jover y López Cerezo, 2008). La innovación social no es por tanto únicamente aquella que tiene a la sociedad en su fin, sino también la que tiene a la sociedad en su origen. Al hablar de innovaciones tecnocientíficas, la innovación social "desde la sociedad" supone la capacidad de quienes no son expertos certificados para producir conocimientos y prácticas que involucran a la ciencia y la tecnología, una suerte de "innovación silvestre" (in the wild), tomando el término con el que Callon y Rabeharisoa (2003) se refieren a la investigación que se realiza fuera de las ubicaciones certificadas y que ejemplifican en la Asociación Francesa de Miopatías.

La innovación social, en sus múltiples formas, ha aparecido así en el panorama sobre la innovación. Pero para reconocer el carácter social que le corresponde a la innovación no es suficiente, en nuestra opinión, extender el significado de esta hasta incluir también los "fines sociales". El desafío no es únicamente ampliar el territorio sino también modificar el modo en que lo cartografiamos. Los propios procesos de innovación son procesos constitutivamente sociales. El problema de base es la visión tradicional de la tecnología que subyace en la literatura especializada en innovación. Un enfoque más adecuado, que da la relevancia adecuada a los rasgos sociales de los procesos de desarrollo tecnológico, incluidas las fases de difusión y uso, ha venido desarrollándose desde mediados de los años 80 del pasado siglo XX bajo el nombre de SCOT (Social Construction of Technology). La idea central del programa SCOT es estudiar empíricamente los artefactos y sistemas tecnológicos mostrando su flexibilidad interpretativa y analizando los mecanismos sociales mediante los que, en determinado contexto histórico y cultural, se cierra tal flexibilidad y se consolidan las formas concretas de tecnología. La metodología procede identificando actores relevantes para el desarrollo de un sistema tecnológico dado, así como los problemas que estos actores se plantean. Diferentes grupos sociales asociarán significados y problemáticas distintas a la misma tecnología, de tal modo que la evolución de su definición, la priorización de problemas a resolver y la estabilización de determinadas soluciones 
para los mismos dependerán del reparto de fuerzas y las estrategias negociadoras. Ejemplos clásicos los encontramos en el estudio del origen de la bicicleta, la baquelita y la bombilla fluorescente (Bijker, 1995; Bijker, Hughes y Pinch, 1987; Bijker y Law, 1992). En la imagen resultante, la tecnología no es un proceso lineal que dependa del desenvolvimiento de una lógica interna de incremento de la eficiencia, y concluya en la difusión y apropiación económica del producto innovador. Constituye más bien un proceso multidireccional de variación y selección que depende crucialmente de entornos sociales; es un proceso social.

En este sentido, una sociedad de la innovación y una cultura de la innovación no pueden solamente atender a factores de mercado, económicos o productivos. La innovación orientada al mercado es también innovación social, y debe atender a valores sociales (del mismo modo que la innovación social puede atender a valores de mercado). No son categorías excluyentes. Más aún, toda innovación es en cierto sentido innovación social: porque está hecha por la sociedad, porque está orientada a la sociedad o porque necesita a la sociedad para tener éxito. No atender al carácter social de los propios procesos de innovación no es despersonalizar dichos procesos y convertirlos en asuntos puramente técnicos que permitan optimizar su aprovechamiento económico. Es más bien transformarlos en cajas negras, y oscurecer intereses y protagonismos; es situarlos más allá del necesario escrutinio público.

\section{REFERENCIAS}

Barnes, B. (1998), The Nature of Power, Cambridge: Polity Press.

Bijker, W. (1995), Of bicycles, bakelites and bulbs: Toward a Theory of Sociotechnical Change, Cambridge, MA: MIT Press.

Bijker, W., T.P. Hughes y T. Pinch (eds.) (1987), The Social Construction of Technological Systems: New Directions in the Sociology and History of Technology. Cambridge, MA: MIT Press.

Bijker, W. y J. Law (eds.) (1992), Shaping Technology/Building Society: Studies in sociotechnical change, Cambridge, MA: MIT Press.

Callon, M. and Rabeharisoa, V. (2003), "Research 'in the wild ' and the shaping of new social identities," Technology in Society 25: 193-204.

Echeverría, J. (2008), "El Manual de Oslo y la innovación social”, Arbor CLXXXIV/732 (julio-agosto): 609-618. 
Edwards-Schachte, M.E., C.E. Matti y E. Alcántara (2012), "Fostering Quality of Life through Social Innovation: A Living Lab Methodology Study Case", Review of Policy Research 29/6: pages 672-692.

Guston, D. (2000), "Retiring the Social Contract for Science", Issues in Science and Technology, verano de 2000.

Mulgan, G., S. Tucker, R. Ali y B. Sanders (2007), Social Innovation: What it is, why it matters and how it can be accelerated. Oxford: Skoll Centre for Social Entrepreneurship.

Núñez Jover, J. y J.A. López Cerezo (2008), “Technological innovation as social innovation: Science, technology, and the rise of STS studies in Cuba", Science, Technology and Human Values 33/6: 707-729.

OECD (1997), National Innovation Systems, París: OECD.

OECD (2005), Oslo Manual: Guidelines for Collecting and Interpreting Innovation Data, $3^{\mathrm{a}}$ ed., París: OECD.

RICYT/OEA/CYTED (2001), Manual de Bogotá: Normalización de Indicadores de Innovación Tecnológica en América Latina y el Caribe. Bogotá: RICYT/OEA/CYTED.

Sabeti, (2009), The Emerging Fourth Sector. Washington, DC: The Aspen Institute.

Sebastián, J. (2000a), “Guía Iberoamericana de la Administración Pública de la Ciencia”, en www.campus-oei.org/guiaciencia/espana.htm (consultado el 7 de diciembre de 2012).

Sebastián, J. (2000b), "Las lógicas de la ciencia y la tecnología en el contexto de la gobernabilidad democrática", Cadernos de Gestâo Tecnológica 47.

Sebastián, J. y E. Muñoz (eds.) (2006), Radiografía de la investigación pública en España. Madrid: Biblioteca Nueva.

Todt, O., M. I. González y B. Estévez (2011), "Conflict in the Sea of Trafalgar: Offshore wind energy and its context", Wind Energy 14/5: 699-706. 